\title{
Fatty Acid Composition of Serum Phospholipids in an Elderly Institutionalized Japanese Population
}

\author{
Ryutaro TaKahashi, * Hideki Ito, and David F. Hor Robin \\ Tokyo Metropolitan Geriatric Hospital (RT, HI), Itabashi-ku, Tokyo, Japan, \\ and The Efamol Research Institute (DFH), PO Box 818, \\ Kentville, Nova Scotia, Canada
}

(Received January 21, 1991)

\begin{abstract}
Summary The fatty acid composition of serum phospholipids of 277 persons, aged 63-97y (average 78y), was measured. Subjects had been living independently in a facility with full food service for $1 \mathrm{y}$ or more. Eleven saturated and unsaturated fatty acids were detected in all the subjects. Stearic acid $(18: 0)$ was positively $(p=0.028)$ and dihomogamma-linolenic acid $(20: 3 n-6)$ was negatively $(p=0.012)$ related to age. Arachidonic acid $(20: 4 n-6)$ tended to decline with age $(p=0.063)$. In addition, the oleic acid (18:1 n-9)/18:0 ratio decreased significantly with age $(p=0.024)$. $18: 0$ and total saturated fatty acids were lower, and linoleic acid (18:2n-6) and 20:4n-6 and n-6 fatty acids were higher in males than in females. These results suggest deficits in $n-6$ essential fatty acids as age advances, particularly in females.
\end{abstract}

Key Words diet, aging, saturated fatty acids, essential fatty acids, dihomo-gamma-linolenic acid, delta-6-desaturase, serum phospholipids

Recent studies in experimental animals have consistently shown that conversion of linoleic acid $(18: 2 n-6)$ to gamma-linolenic acid $(18: 3 n-6)$, which is mediated by the enzyme delta-6-desaturase and is the rate-limiting step of $n-6$ essential fatty acid (EFA) metabolism, is lowered as the animal's age advances (13). However, no evidence yet exists about this in humans, in part due to difficulty in providing constant dietary conditions. Although EFA metabolism is affected by hormonal (insulin, glucagon, etc.) and dietary (protein, caloric restriction, etc.) factors, the fatty acid content of the diet is a major determinant of fatty acid composition of the blood $(4,5)$, especially for those fatty acids actually found in the diet. While linoleic acid alone (the parent fatty acid of the $n-6$ EFAs) can be provided in the diet of experimental animals, it is almost impossible to do this in

* Address reprint requests to: Dr. R. Takahashi, Endocrinology and Metabolism Section, Tokyo Metropolitan Geriatric Hospital, 35-2 Sakaecho, Itabashi-ku, Tokyo 173, Japan. 
humans, as omnivores and meat eaters acquire a good deal of arachidonic acid (20: $4 n-6$, the main metabolite of $n-6$ EFAs) and other EFAs, as well as linoleic acid, in their food. This may be an important factor which contributes to differences between human studies and animal experiments. A desirable approach when examining changes of EFAs with age would be to obtain blood samples from people living in a facility with a food service and who are thus all taking approximately the same diet. We had the opportunity to carry out this kind of clinical study in an institutionalized population containing individuals over a wide age range.

\section{SUBJECTS AND METHODS}

From May 31, 1989 to June 2, 1989, a regular health checkup was conducted on 449 people living in a facility which is located on the grounds of the Tokyo Metropolitan Geriatric Hospital. This facility is for elderly people (aged 63-97 y) and provides a full food service. All the individuals were independent with regard to daily activities and free of terminal, wasting illness, untreated diabetes mellitus, or active liver disease. As people who had lived in the institution for less than $1 \mathrm{y}$ were excluded from the study, all the subjects had consumed similar meals each day for $1 \mathrm{y}$ or more. Forty people, taken from the whole population, were interviewed to examine how often they took meals outside the institution. Twenty-seven people did not eat outside and even the others took at least two of their three meals each day at the institution. These others usually took supper or lunch once or twice per week outside. The main foods eaten were sushi (raw fish on rice ball), unadon (buked eel), zaru-soba (buckwheat noodles), and tempura (fish and vegetables fried in oil), which all are typical dishes for Japanese taking meals in restaurants. No individual went outside for meals every day, mainly for economic reasons. Blood samples were taken from all individuals: for 277 (119 males and 158 females), excess serum that remained after laboratory tests was available and sufficient for the present study.

Menus for 7 consecutive days before the study were obtained from the institution. Foods and beverages were analyzed for nutrient composition using the Standard Tables of Food Composition in Japan (4th edition, Science and Technology Agency, Japan, 1982). Table 1 summarizes the dietary data. Guidelines for healthy American adults issued by the American Heart Association are also shown as a reference (6). The institutional menus were well within these guidelines. Serum lipids were extracted with chloroform : methanol $(2: 1)$ containing $0.02 \%$ butylated hydroxytoluene as antioxidant, and phospholipid fractions were separated by thin-layer chromatography using a solvent system of petroleum ether: diethyl ether : acetic acid:methanol, $85: 15: 2.5: 1$ (by vol). Methyl esters were prepared by treatment of samples with $14 \%$ boron trifluoride in methanol and relative fatty acid composition was determined by gas-liquid chromatography as previously described (7).

Results are shown as means \pm SD. Sex differences in each age group were 
Table 1. Composition of meals consumed during 7 consecutive days before the study.

\begin{tabular}{lccc}
\hline \multicolumn{1}{c}{ Per day } & \multicolumn{2}{c}{ Means \pm SD (range) } & AHA guideline ${ }^{\mathrm{a}}$ \\
\hline Total kcal & $1,860 \pm 121$ & $(1,632-2,023)$ & \\
Total fat $(\mathrm{g})$ & $38.4 \pm 8.3$ & $(22.5-47.6)$ & \\
$\quad$ (\% of total kcal) & $18.6 \pm 3.3$ & $(12.4-22.6)$ & less than $30 \%$ \\
Polyunsaturated fat $(\mathrm{g})$ & $11.8 \pm 3.5$ & $(7.4-18.1)$ & \\
(\% of total kcal) & $5.7 \pm 1.6$ & $(4.1-8.1)$ & less than $10 \%$ \\
Saturated fat $(\mathrm{g})$ & $10.0 \pm 3.7$ & $(6.8-18.0)$ & \\
$\quad$ (\% of total kcal) & $4.8 \pm 1.7$ & $(3.7-8.0)$ & less than $10 \%$ \\
P/S ratio & $1.19 \pm 0.28$ & $(0.98-1.85)$ & \\
Total cholesterol $(\mathrm{mg})$ & $249 \pm 90$ & $(120-412)$ & less than $300 \mathrm{mg}$ \\
Carbohydrate $(\mathrm{g})$ & $301 \pm 15$ & $(280-322)$ & \\
$\quad$ (\% of total kcal) & $64.7 \pm 4.0$ & $(57.8-70.5)$ & more than $50 \%$ \\
\hline
\end{tabular}

${ }^{a}$ Dietary guidelines for healthy American adults issued by the American Heart Association (AHA), taken from reference (6).

analyzed by Student's $t$-test. Pearson's correlation coefficient was used to measure the linear relation between age and proportional concentration of each fatty acid. Differences were considered significant at $p<0.05$.

\section{RESULTS}

The fatty acid compositions of males and females are shown in Table 2, with each sex divided into 5 groups according to age ( 60 to 69,70 to 74,75 to 79,80 to 84 and over 85 years old). There were substantial sex differences: stearic acid (18: 0 ) was lower and linoleic acid and arachidonic acid were significantly higher in males than in females in each age group with few exceptions. In addition, the total of saturated fatty acids $(14: 0+16: 0+18: 0)$ was significantly lower in males than in females except for the 60- to 69-year-old group, while that of $n$-6 unsaturated fatty acids $(18: 2+18: 3+20: 2+20: 3+20: 4+22: 4+22: 5)$ was significantly higher in males than in females in all the age groups. However, there was no consistent gender difference with regard to the $n-3$ fatty acids.

Correlations between age and proportions of 11 saturated and unsaturated fatty acids were computed. Stearic acid showed a significant positive correlation (Fig. 1), and dihomo-gamma-linolenic acid (20:3n-6) showed a significant negative correlation (Fig. 2). Although arachidonic acid showed a trend of decline with age $(p=0.063)$, there were no significant relationships for the other fatty acids. Correlations between age and the ratio of oleic acid to stearic acid were also calculated and reached statistical significance $(p=0.024)$. Ratios of post-delta-6desaturase $n-6$ fatty acids $(18: 3+20: 3+20: 4+22: 4+22: 5)$ to linoleic acid and post-delta-6-desaturase $n-3$ fatty acids $(20: 5+22: 5+22: 6)$ to alfa-linolenic acid $(18: 3 n-3)$ did not change as age advanced. 
Table 2. Fatty acid composition of serum phospholipids in the institutionalized population.

\begin{tabular}{|c|c|c|c|c|c|c|}
\hline & \multicolumn{6}{|c|}{ Fatty acids $(\%)$} \\
\hline & Age & Number & $16: 0$ & $16: 1 n-7$ & $18: 0$ & $18: 1 n-9$ \\
\hline \multirow{7}{*}{ Male } & $60-69$ & 25 & $29.4 \pm 2.0$ & $1.35 \pm 0.62$ & $8.2 \pm 1.9$ & $10.3 \pm 1.7$ \\
\hline & $70-74$ & 22 & $28.6 \pm 2.0$ & $1.27 \pm 0.67$ & $8.0 \pm 1.6$ & $10.3 \pm 2.6$ \\
\hline & $75-79$ & 29 & $29.1 \pm 1.9$ & $1.37 \pm 0.99$ & $8.2 \pm 1.9$ & $10.0 \pm 1.6$ \\
\hline & $80-84$ & 18 & $28.7 \pm 1.9$ & $0.95 \pm 0.26$ & $8.1 \pm 2.1$ & $9.7 \pm 1.9$ \\
\hline & $85^{-}$ & 25 & $28.7 \pm 1.8$ & $1.37 \pm 0.66$ & $8.7 \pm 2.0$ & $9.8 \pm 1.6$ \\
\hline & Total & 119 & $28.9 \pm 1.9$ & $1.29 \pm 0.72$ & $8.0 \pm 1.7$ & $10.0 \pm 1.8$ \\
\hline & Number & & 119 & 115 & 119 & 119 \\
\hline \multirow{7}{*}{ Female } & $60-69$ & 10 & $30.0 \pm 2.4$ & $1.51 \pm 0.78$ & $9.8 \pm 1.9 *$ & $10.3 \pm 1.6$ \\
\hline & $70-74$ & 28 & $29.4 \pm 1.1$ & $1.22 \pm 0.45$ & $11.0 \pm 2.4^{*}$ & $10.3 \pm 1.5$ \\
\hline & $75-79$ & 28 & $29.5 \pm 1.5$ & $1.16 \pm 0.41$ & $10.8 \pm 2.1^{*}$ & $10.5 \pm 1.6$ \\
\hline & $80-84$ & 50 & $29.3 \pm 1.2$ & $1.18 \pm 0.35$ & $10.9 \pm 2.1^{*}$ & $10.2 \pm 1.1$ \\
\hline & $85-$ & 42 & $29.7 \pm 1.5$ & $1.21 \pm 0.40$ & $10.5 \pm 2.1^{*}$ & $10.7 \pm 1.8$ \\
\hline & Total & 158 & $29.5 \pm 1.4$ & $1.21 \pm 0.43$ & $10.7 \pm 2.1^{*}$ & $10.4 \pm 1.5$ \\
\hline & Number & & 158 & 146 & 158 & 158 \\
\hline
\end{tabular}

Table 2. (continued).

\begin{tabular}{clcccc}
\hline & \multicolumn{5}{c}{ Fatty acids (\%) } \\
\cline { 2 - 6 } & \multicolumn{1}{c}{ Age } & Number & $18: 2 n-6$ & $18: 3 n-3$ & $20: 3 n-6$ \\
\hline \multirow{6}{*}{ Male } & $60-69$ & 25 & $23.0 \pm 3.8$ & $0.35 \pm 0.12$ & $2.07 \pm 0.42$ \\
& $70-74$ & 22 & $23.5 \pm 3.7$ & $0.32 \pm 0.09$ & $1.97 \pm 0.52$ \\
& $75-79$ & 29 & $22.9 \pm 4.0$ & $0.31 \pm 0.11$ & $2.12 \pm 0.57$ \\
& $80-84$ & 18 & $24.2 \pm 3.4$ & $0.29 \pm 0.06$ & $1.74 \pm 0.56$ \\
& $85-$ & 25 & $23.1 \pm 4.5$ & $0.36 \pm 0.09$ & $1.85 \pm 0.46$ \\
& Total & 119 & $23.3 \pm 3.9$ & $0.33 \pm 0.10$ & $1.97 \pm 0.52$ \\
& Number & & 119 & 95 & 119 \\
\hline \multirow{6}{*}{ Female } & $60-69$ & 10 & $18.6 \pm 3.5^{*}$ & $0.31 \pm 0.06$ & $2.14 \pm 0.50$ \\
& $70-74$ & 28 & $20.0 \pm 2.9^{*}$ & $0.31 \pm 0.07$ & $2.16 \pm 0.57$ \\
& $75-79$ & 28 & $20.3 \pm 2.9^{*}$ & $0.31 \pm 0.08$ & $2.28 \pm 0.68$ \\
& $80-84$ & 50 & $19.9 \pm 4.2^{*}$ & $0.31 \pm 0.07$ & $2.26 \pm 0.58^{*}$ \\
& $85-$ & 42 & $20.0 \pm 3.1^{*}$ & $0.32 \pm 0.08$ & $1.79 \pm 0.54$ \\
& Total & 158 & $20.0 \pm 3.4^{*}$ & $0.31 \pm 0.07$ & $2.12 \pm 0.61$ \\
& Number & & 158 & 134 & 158 \\
\hline
\end{tabular}

\section{DISCUSSION}

The present study indicates changes of fatty acid composition as age progresses and also with sex in clearly defined dietary conditions: (1) all the subjects were institutionalized and had been provided with a full food service for at least $1 \mathrm{y}$; and 
Table 2. (continued).

\begin{tabular}{|c|c|c|c|c|c|c|}
\hline & \multicolumn{6}{|c|}{ Fatty acids $(\%)$} \\
\hline & Age & Number & $20: 4 n-6$ & $20: 5 n-3$ & $22: 5 n-3$ & $22: 6 n-3$ \\
\hline \multirow{7}{*}{ Male } & $60-69$ & 25 & $10.4 \pm 2.0$ & $3.5 \pm 1.3$ & $1.08 \pm 0.33$ & $9.7 \pm 2.4$ \\
\hline & $70-74$ & 22 & $10.3 \pm 1.5$ & $3.6 \pm 1.4$ & $1.17 \pm 0.35$ & $10.3 \pm 2.3$ \\
\hline & $75-79$ & 29 & $10.7 \pm 2.0$ & $3.7 \pm 1.2$ & $1.14 \pm 0.33$ & $10.0 \pm 2.0$ \\
\hline & $80-84$ & 18 & $10.8 \pm 2.0$ & $4.2 \pm 1.6$ & $0.99 \pm 0.27$ & $9.6 \pm 1.2$ \\
\hline & $85^{-}$ & 25 & $9.9 \pm 1.8$ & $3.7 \pm 1.1$ & $1.17 \pm 0.32$ & $11.0 \pm 2.5$ \\
\hline & Total & 119 & $10.4 \pm 1.9$ & $3.7 \pm 1.3$ & $1.12 \pm 0.32$ & $10.2 \pm 2.2$ \\
\hline & Number & & 119 & 119 & 94 & 119 \\
\hline \multirow{7}{*}{ Female } & $60-69$ & 10 & $10.0 \pm 1.9$ & $3.8 \pm 1.2$ & $1.24 \pm 0.20$ & $10.9 \pm 1.8$ \\
\hline & $70-74$ & 28 & $8.9 \pm 1.7^{*}$ & $3.6 \pm 1.1$ & $1.18 \pm 0.26$ & $10.4 \pm 1.6$ \\
\hline & $75-79$ & 28 & $9.4 \pm 2.0^{*}$ & $3.3 \pm 0.9$ & $1.14 \pm 0.31$ & $9.9 \pm 1.9$ \\
\hline & $80-84$ & 50 & $9.2 \pm 1.4^{*}$ & $3.6 \pm 1.3$ & $1.20 \pm 0.32 *$ & $10.4 \pm 1.9$ \\
\hline & $85-$ & 42 & $9.5 \pm 1.7$ & $3.8 \pm 1.1$ & $1.15 \pm 0.31$ & $10.3 \pm 1.8$ \\
\hline & Total & 158 & $9.3 \pm 1.7 *$ & $3.6 \pm 1.1$ & $1.17 \pm 0.30$ & $10.3 \pm 1.8$ \\
\hline & Number & & 158 & 158 & 152 & 158 \\
\hline
\end{tabular}

Table 2. (continued).

\begin{tabular}{|c|c|c|c|c|c|}
\hline & \multicolumn{5}{|c|}{ Fatty acids $(\%)$} \\
\hline & Age & Number & Saturates ${ }^{\mathrm{a}}$ & $n-6$ unsaturates $^{\mathrm{b}}$ & $n-3$ unsaturates $^{\mathrm{c}}$ \\
\hline \multirow{7}{*}{ Male } & $60-69$ & 25 & $39.1 \pm 7.0$ & $35.8 \pm 4.6$ & $14.4 \pm 3.7$ \\
\hline & $70-74$ & 22 & $37.1 \pm 3.3$ & $35.7 \pm 4.0$ & $15.3 \pm 3.6$ \\
\hline & $75-79$ & 29 & $37.4 \pm 3.2$ & $36.0 \pm 4.3$ & $14.8 \pm 3.0$ \\
\hline & $80-84$ & 18 & $37.6 \pm 2.5$ & $36.9 \pm 3.5$ & $14.6 \pm 2.2$ \\
\hline & $85-$ & 25 & $37.1 \pm 2.4$ & $35.3 \pm 4.9$ & $16.0 \pm 3.5$ \\
\hline & Total & 119 & $37.7 \pm 4.1$ & $35.8 \pm 4.3$ & $15.0 \pm 3.3$ \\
\hline & Number & & 119 & 119 & 119 \\
\hline \multirow{7}{*}{ Female } & $60-69$ & 10 & $39.2 \pm 2.8$ & $31.2 \pm 4.2^{*}$ & $16.3 \pm 2.5$ \\
\hline & $70-74$ & 28 & $40.6 \pm 2.6^{*}$ & $32.0 \pm 3.3^{*}$ & $15.2 \pm 2.5$ \\
\hline & $75-79$ & 28 & $40.6 \pm 2.6^{*}$ & $32.6 \pm 3.2^{*}$ & $14.6 \pm 2.9$ \\
\hline & $80-84$ & 50 & $40.5 \pm 2.5^{*}$ & $32.1 \pm 4.4^{*}$ & $15.5 \pm 3.0$ \\
\hline & $85-$ & 42 & $40.5 \pm 2.4^{*}$ & $32.0 \pm 3.0^{*}$ & $15.6 \pm 3.0$ \\
\hline & Total & 158 & $40.4 \pm 2.5^{*}$ & $32.1 \pm 3.6^{*}$ & $15.4 \pm 2.9$ \\
\hline & Number & & 158 & 158 & 158 \\
\hline
\end{tabular}

Results given are means $\pm \mathrm{SD}$. $*_{p}<0.05$ or better in comparison with the corresponding male group. ${ }^{\mathrm{a}}$ Saturates $=14: 0+16: 0+18: 0,{ }^{\mathrm{b}} n-6$ unsaturates $=$ $18: 2+18: 3+20: 2+20: 3+20: 4+22: 4+22: 5,{ }^{c} n-3 \quad$ unsaturates $=18: 3+20: 5+$ $22: 5+22: 6$.

(2) fat composition and cholesterol content (Table 1) are at the same levels as those of the 1960s Japanese diet (8) and were well within the guidelines for healthy adults in the United States (6). Although the subjects were limited to elderly people, all of 


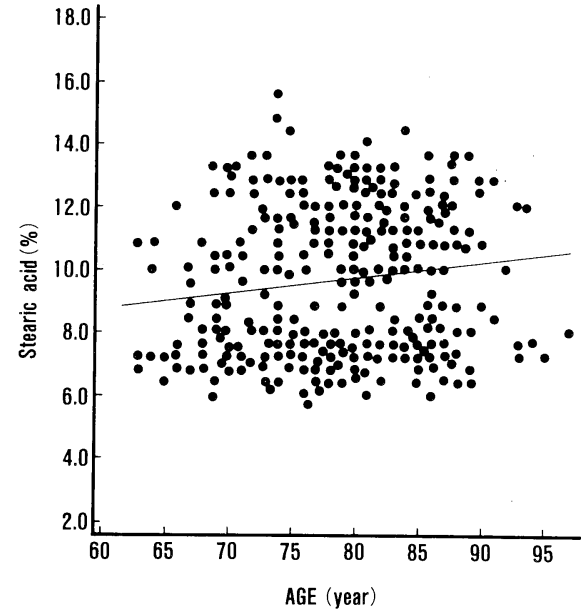

Fig. 1

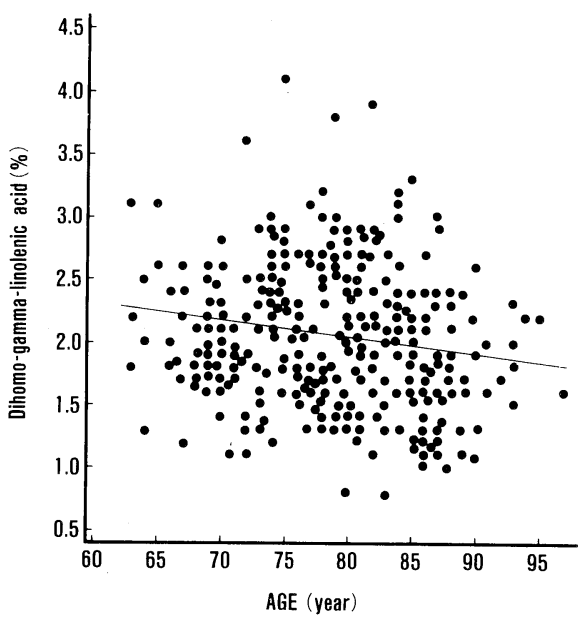

Fig. 2

Fig. 1. Age-dependent changes in stearic acid in serum phospholipids. $r=0.115$ and $p=0.028$.

Fig. 2. Age-dependent changes in dihomo-gamma-linolenic acid in serum phospholipids of the institutionalized population. $r=-0.136$ and $p=0.012$.

whom did not take all their meals in the institution, the age range was relatively wide (63 to 97 years old) and the nutritional intake as constant as is ever likely to be obtained.

In a study of individuals from 0 to 90 years old, but without dietary control, Holman et al. reported that total serum lipid unsaturation was significantly higher in males than in females (9). However, no individual fatty acid showed a statistically significant difference between the sexes. Another study of healthy volunteers also showed no sex difference (7). In contrast, the present study clearly indicated that stearic acid and total saturated fatty acids are lower and linoleic acid, arachidonic acid, and total $n-6$ fatty acids are higher in elderly Japanese males than in females; these results were consistently shown in all the age groups with few exceptions and seem to be in disagreement with studies in experimental animals (10, 11). Female rats are less sensitive to EFA deficiency than male rats and maintain higher concentrations of linoleic acid in adipose tissue (10). Reduction of postdelta-6-desaturase products by dietary cholesterol administration is significantly greater in male rats than in females (11). Thus considerable caution is required when extrapolating to humans results of male-female difference of EFAs metabolism obtained in experimental animals.

It has been hypothesized that in humans levels of dihomo-gamma-linolenic acid may decline with aging primarily because of reduced delta-6-desaturase activity (12). Although arachidonic acid is also a metabolite of linoleic acid, it is less likely to decline with aging as omnivore humans obtain important amounts of 


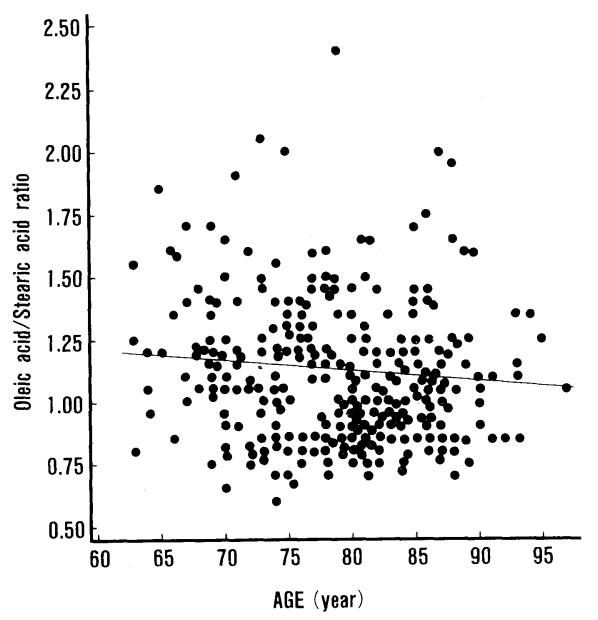

Fig. 3. Age-dependent changes in oleic acid/stearic acid ratio in serum phospholipids of the institutionalized population. $r=-0.119$ and $p=0.024$.

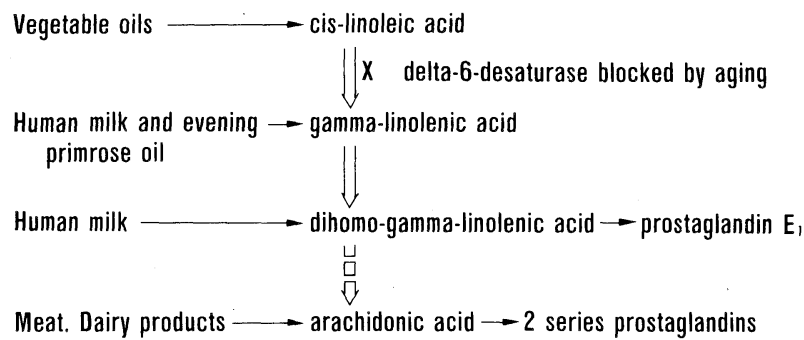

Fig. 4. An outline of the pathway of essential fatty acid metabolism and the food sources of the various items.

arachidonic acid in the diet from meat (13) and fish. Gamma-linolenic acid, the immediate delta-6-desaturase metabolite of linoleic acid, is found in plasma only in small amounts because it is easily elongated to dihomo-gamma-linoleic acid. Consequently, a deficiency of EFAs with aging is likely to appear as a decline of dihomo-gamma-linolenic acid rather than arachidonic acid (Fig. 4). As our preliminary data showed age-related reduction of levels of plasma total phospholipids, deficiency of dihomo-gamma-linolenic acid is expected to be much greater (data not shown).

Dihomo-gamma-linolenic acid deficiency with age may directly cause reductions of levels of prostaglandin E1 and of 15-hydroxy-dihomo-gamma-linolenic acid (14), which possess important actions on the cardiovascular system and on inflammation (15). According to the statistics in Japan, the predominant cause of death over $80 \mathrm{y}$ is cardiovascular disease and the peak ages of morbidity from rheumatoid arthritis and asthma are $75-79 y$ and $80-84 \mathrm{y}$, respectively $(16,17)$. 
Wood et al. reported low levels of dihomo-gamma-linolenic acid in adipose tissue in men with new coronary heart disease (18). In a prospective study, low dihomogamma-linolenic acid levels in cholesterol esters, triglycerides, and phospholipids also seem to be associated with a risk of stroke (19), which is a high prevalent vascular disorder in Japan (16). Eskimos, who have a very low prevalence of cardiovascular disease, rheumatoid arthritis, and asthma, indicate unusually high concentrations of this fatty acid in plasma (20). This suggests a relationship between age-dependent pathological conditions and dihomo-gamma-linolenic acid deficiency (12).

The decrease of the oleic acid/stearic acid ratio with age may be due to an increase of stearic acid (Fig. 1), possibly due to decrease in the aging process of delta-9-desaturase, which converts stearic acid to oleic acid. This enzyme is activated in the EFAs' deficient states and triggers the production of eicosatrienoic acid $(20: 3 n-9)(21)$.

Thus, these age-related changes of fatty acids (at least at ages $60 \mathrm{y}$ and over) lead to a decrease of total unsaturation products, which may directly or indirectly through alterations of cholesterol metabolism (22) affect membrane lipid configuration. Receptor loss and lipid peroxidation with age (23) might be explained by this.

We thank Dr. Yujiro Suzuki, Tokyo Metropolitan Geriatric Hospital, for help with serum sample selection; Ms. Nancy Morse-Fisher, Chief Technician, Efamol Research Institute, for help with gas-liquid chromatography analyses; and Ms. Fujiko Fujikake, Nutritionist, Tokyo Metropolitan Geriatric Hospital, for help with dietary records assessment.

\section{REFERENCES}

1) Ayara, S., Gaspar, G., Brenner, R. R., Peluffo, R. O., and Kunau, W. (1973): Fate of linoleic, arachidonic, and docosa-7,10,13,16-tetraenoic acids in rat testicles. J. Lipid Res., 14, 296-305.

2) Bordoni, A., Biagi, P. L., Turchetto, E., and Hrelia, S. (1988): Aging influence on delta-6-desaturasé activity and fatty acid composition of rat liver microsomes. Biochem. Int., 17, 1001-1009.

3) Hrelia, S., Bordoni, A., Celadon, M., Turchetto, E., Biagi, P. L., and Rossi, C. A. (1989): Age-related changes in linoleate and $\alpha$-linolenate desaturation by rat liver microsomes. Biochem. Biophys. Res. Commun., 163, 348-355.

4) Holman, R. T. (1986): Control of polyunsaturated acids in tissue lipids. J. Am. Coll. Nutr., 5, 183-211.

5) Tilvis, R. S., and Miettinen, T. A. (1985): Fatty acid compositions of serum lipids, erythrocytes, and platelets in insulin-dependent diabetic women. J. Clin. Endocrinol. \& Metab., 61, 741-745.

6) Dietary guidelines for healthy American adults. A statement for physicians and health professionals by the nutrition committee, American Heart Association (1988): Arte- 
riosclerosis, 8, 218A-221A.

7) Manku, M. S., Horrobin, D. F., Huang, Y.-S., and Morse, N. (1983): Fatty acids in plasma and red cell membranes in normal humans. Lipids, 18, 906-908.

8) Wen, C.-P., and Gershoff, S. N. (1973): Changes in serum cholesterol and coronary heart disease mortality associated with changes in the postwar Japanese diet. Am. J. Clin. Nutr., 26, 616-619.

9) Holman, R. T., Smythe, L., and Johnson, S. (1979): Effect of sex and age on fatty acid composition of human serum lipids. Am. J. Clin. Nutr., 32, 2390-2399.

10) Ostwald, R., Bouchard, P., Miljanich, P., and Lyman, R. L. (1965): Influence of sex and gonadal hormones on rat liver and carcass lipids during the development of an. essential fatty acid deficiency. Biochem. J., 97, 485-499.

11) Huang, Y.-S., Mitchell, J., Manku, M. S., and Horrobin, D. F. (1985): Effect of cholesterol feeding and sex difference on the tissue n-6 and n-3 fatty acid levels in fat-deficient rats treated with linoleate or linolenate. Nutr. Res., 5, 535-543.

12) Horrobin, D. F. (1981): Loss of delta-6-desaturase activity as a key factor in aging. Med. Hypotheses, 7, 1211-1220.

13) Takahashi, R., Morita, I., Murota, S., Shiraki, M., Ito, H., and Orimo, H. (1983): Dietary arachidonic acid supplementation increases thromboxane synthesizing activity in platelets from diabetics. Prostaglandins Leukotrienes Med., 11, 443-450.

14) Miller, C. C., McCreedy, C. A., Jones, A. D., and Ziboh, V. A. (1988): Oxidative metabolism of dihomogammalinolenic acid by guinea pig epidermis: evidence of generation of anti-inflammatory products. Prostaglandins, 35, 917-938.

15) Horrobin, D. F. (1988): Prostaglandin $E_{1}$ : physiological significance and clinical use. Wien. Klin. Wochenschr., 10, 471-477.

16) Ministry of Health and Welfare, Japan (1986): Population Movement Statistics.

17) Ministry of Health and Welfare, Japan (1984): General Condition of Patient Survey.

18) Wood, D. A., Butler, S., Riemersma, R. A., Thomson, M., and Oliver, M. F. (1984): Adipose tissue and platelet fatty acids and coronary heart disease in Scottish men. Lancet, ii, 117-121.

19) Miettinen, T. A., Huttunen, J. K., and Naukkarinen, V. (1986): Cholesterol and fatty acids of serum lipids as risk factors of stroke. Monogr. Atheroscler., 14, 19-25.

20) Horrobin, D. F. (1987): Low prevalences of coronary heart disease (CHD), psoriasis, asthma and rheumatoid arthritis in Eskimos: are they caused by high dietary intake of eicosapentaenoic acid (EPA), a genetic variation of essential fatty acid (EFA) metabolism or a combination of both? Med. Hypotheses, 22, 421-428.

21) Mead, J. F. (1970): The metabolism of the polyunsaturated fatty acids. Prog. Chem. Fats Other Lipids, 9, 159-192.

22) Sinclair, H. M. (1990): History of essential fatty acids, in Omega-6 Essential Fatty Acids: Pathophysiology and Roles in Clinical Medicine, ed. by Horrobin, D. F., Alan Liss, New York, pp. 1-20.

23) Schroeder, F. (1984): Role of membrane lipid asymmetry in aging. Neurobiol. Aging, 5, 323-333. 